\title{
Stratigraphie der jüngeren Pyroklastika der Sierra Nevada de México durch schwermineralanalytische und pedologische Untersuchungen 1)
}

\author{
Von Günter Miehlich, Reinbek \\ Mit 6 Abbildungen und 5 Tabellen
}

Z u s a m m e n f s s u ng. Zur Überprüfung der makroskopisch erarbeiteten Stratigraphie
der jüngeren Pyroklastika der Sierra Nevada de México wurden schwermineralanalytische und
pedologische Untersuchungen an mehr als 200 Einzelproben durchgeführt. Die Schwermineralver-
hältnisse sind ungeeignet zur Unterscheidung von Aschenlagen, die sich makroskopisch nicht aus-
inanderhalten lassen. Sie sind entweder zu ähnlich, oder sie streuen innerhalb einer Schicht sehr
tark, was v. a. auf eine Sortierung der Aschen in Abhängigkeit zur Entfernung vom Krater zu-
tückgeführt werden kann.

Zur Stratigraphie geeignete Bodenmerkmale müssen eine monotone Altersfunktion und eine geringe Tiefenfunktion haben sowie verlagerungsresistent sein. Darüber hinaus muß der Einfluß des Alters auf die Bodeneigenschaften größer sein als die differenzierende Wirkung der übrigen Faktoren der Bodenbildung.

Der Gehalt an dithionitlöslichem Eisen und der Aktivitätsgrad pedogenen Eisens ermöglicht die Unterscheidung der makro-, mikroskopisch und schwermineralanalytisch nicht unterscheidbaren Pyroklastika.

S u m mary. Heavy mineral analytic and soil tests with more than 200 samples were used for verifying a macroscopically elaborated stratigraphy of the more recent pyroclasts of the Sierra Nevada de México. Heavy mineral relationships are not suitable for the differentiation of ash layers, which cannot be separated macroscopically. They are either too similar or variation within one layer is very strong, which may be attributed primarily to sorting of ashes in relation to the distance from the crater.

Soil properties suitable for stratigraphy must have a monotonous age function and a weak depth function, in addition to showing no translocation. Furthermore, the influence of age on soil properties must exeed the differentiating effect of the remaining factors of soil formation.

While a difference between pyroclasts could be established neither macroscopically, microscopically nor by heavy mineral analysis, alone the content of dithionite-soluble iron and the relation between oxalate-soluble and dithionite-soluble iron permitted a differentation of pyroclasts.

\section{Einleitung}

Die Bodenbildung in der Sierra Nevada de México wird neben dem Klima dominierend von Eigenschaften und Alter der Ausgangsgesteine bestimmt. Eine Voraussetzung für pedologische Untersuchungen (Kneib, Miehlich \& ZöTtl 1973; Miehlich 1974) in diesem Gebiet war daher die Klärung der Stratigraphie der anstehenden Pyroklastika der Sierra Nevada und die Kartierung der Schüttungsareale der einzelnen Straten.

Unter Berücksichtigung früherer, meist punkthaft angelegter Arbeiten (White 1962, Mooser 1967, Cornwall 1968, Heine \& Heide-Weise 1973, Heine 1973, Heine \& SchöNhals 1973) konnten durch Auswertung von 350 makroskopischen Profilbeschreibungen die Grundzüge der Stratigraphie der jüngeren Pyroklastika und ihrer Verbreitung geklärt werden (KNeib 1973; Miehlich, KNeib \& Heide-Weise 1974). Sie sollen gekürzt zur Einführung in das Arbeitsgebiet den laboranalytischen Untersuchungen vorangestellt werden.

1) Die Untersuchung wurde durch eine Sachbeihilfe der Deutschen Forschungsgemeinschaft im Rahmen des Mexiko-Projekts ermöglicht. Der Verfasser dankt für die großzügige Unterstützung. 

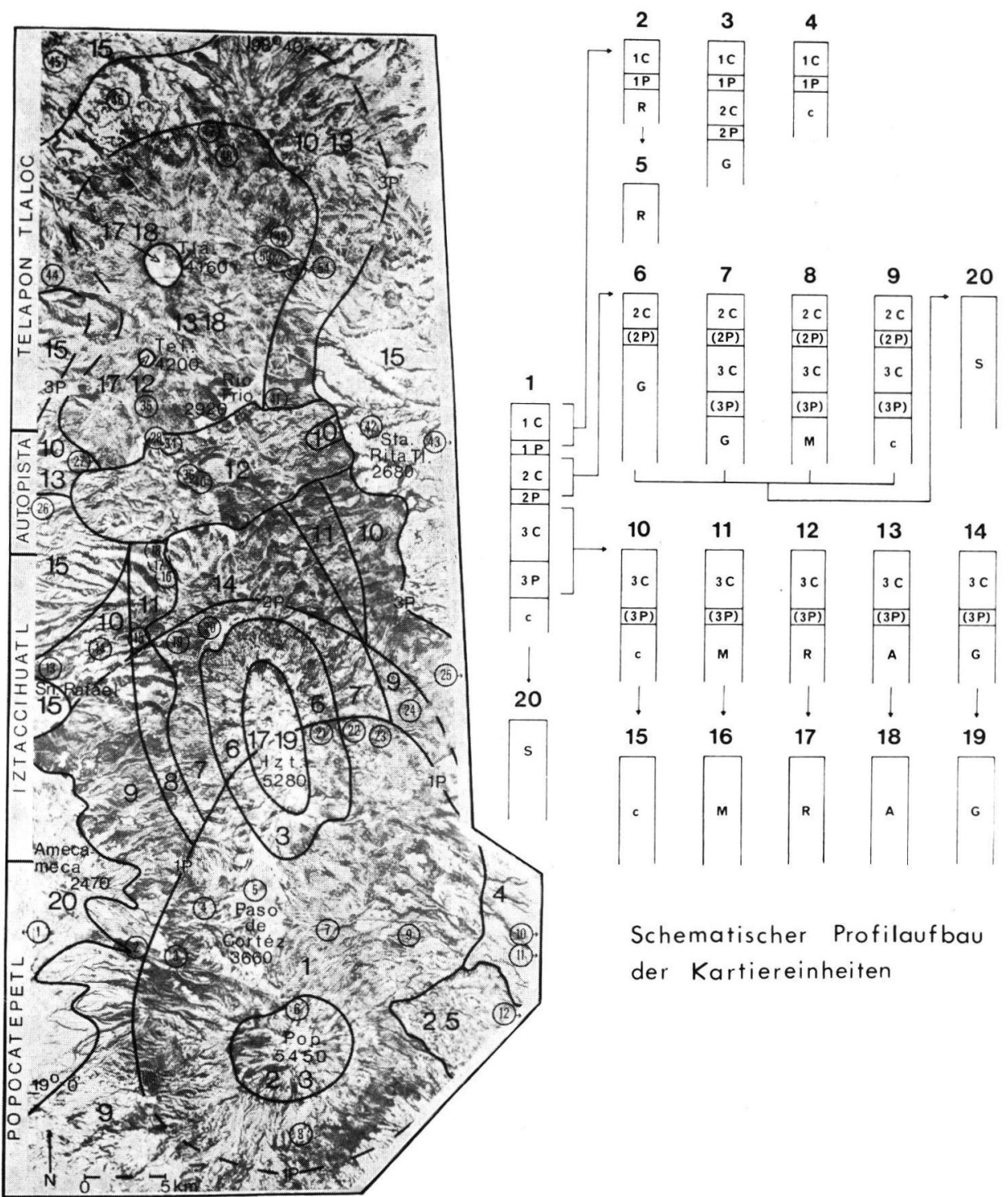

Schematischer Profilaufbau der Kartiereinheiten

\begin{tabular}{|l|c|c|c|c|c|c|c|c|c|c|c|c|c|c|c|c|c|c|c|c|}
\hline $\begin{array}{l}\text { Leitprofile } \\
\text { der Karte }\end{array}$ & 1 & $\begin{array}{l}2 \\
3\end{array}$ & $\begin{array}{l}2 \\
5\end{array}$ & 3 & 4 & 6 & 7 & 8 & 9 & 10 & 10 & 11 & 12 & 13 & 14 & 15 & 17 & 17 & 17 & 20 \\
12 & 18 & 19 & \\
\hline $\begin{array}{l}\text { Begleit - } \\
\text { profile }\end{array}$ & 17 & 17 & & 17 & 15 & 17 & 8 & 9 & 10 & 15 & 18 & 10 & 17 & 19 & 11 & 20 & & & 2 & 15 \\
& 1 & & & 20 & 19 & 16 & & 15 & & & & 10 & 20 & 16 & & & & & \\
20 & & & & & & & & & & & & & & & & & & & & \\
\hline
\end{tabular}

Abb. 1. Verbreitung der jüngeren Pyroklastika der Sierra Nevada de México. Die Kartiereinheiten (große Ziffern) wurden nach der Verbreitung von ein oder zwei flächenmäßig dominierenden Schichtabfolgen abgegrenzt, deren Aufbau dem Schema entnommen werden kann. Die Tabelle zeigt die innerhalb der Kartiereinheiten untergeordnet auftretenden Schichtabfolgen. Schichten in Klammern fehlen teilweise. Die Zahlen in Kreisen bezeichnen Nr. und Lage der entnommenen Profile. Erläuterung der Schichtbezeichnungen im Schema vgl. Text. 
Die Eruptiva der letzten drei großen Ausbruchsphasen des Popocatépetl bedecken den gesamten Bereich der Sierra Nevada de México. Während jeder Eruptionsphase wurde eine häufig mehrteilig zusammengesetzte Bimslage (P) und eine makroskopisch und mikroskopisch nicht weiter unterteilbare Aschenlage (C) gefördert, die mit dem jüngsten Ausbruch beginnend mit 1C, 1P, 2C, 2P, 3C und 3P bezeichnet werden (eine Gliederung, die sich auch HeIne 1973, zu eigen machte; 3P $=$ PWA in Mooser 1967).

Nach den bislang vorliegenden Datierungen haben die Straten folgendes Alter vor heute: Die jüngste Aschenlage 1C ca. 400 Jahre (YARzA 1971), 1P zwischen ca. 400 und ca. 1000 Jahre $^{2}$ ), 2C zwischen ca. 1000 und 4800 Jahre (HeIne 1973), 3C zwischen ca. 4800 und 12000 Jahre, 3P zwischen ca. 12000 und ca. 14700 Jahre.

Die Eruptiva der drei letzten Ausbruchsphasen des Popocatépetl bedecken unterschiedliche Areale (Abb. 1), so daß neben der vollständigen Förderfolge (Nr. 1 im Schema der Abb. 1) Bereiche auftreten, in denen eine oder mehrere Eruptionsphasen fehlen (2, 3, 4, 6, 7, 8, 9, 10, 11, 12, 13 und 14 in Abb. 1).

Das Liegende der jüngeren Pyroklastika des Popocatépetl wird durch Festgestein (R) unterschiedlicher Zusammensetzung (Andesit, Latiandesit, Dazit, Rhyodazit, Alkalirhyolith, frdl. schriftl. Mittlg. Prof. Dr. R. Weyl, Gießen), Gesteinszersatz aus Phänoandesiten (M), phänorhyolithischen vulkanischen Sanden des Tlaloc/Telapón-Gebiets (A), glazial aufbereiteten Pyroklastika und Gesteinen (G) und älteren Pyroklastika (c) gebildet. Da noch während der Eruption der jüngeren Pyroklastika Gletschervorstöße stattfanden (White 1962, Heine 1973) ist in G teilweise Material der jüngeren Pyroklastika 1C bis 3P eingearbeitet. Unter der Bezeichnung $\mathrm{c}$ wurden alle älteren Pyroklastika einschließlich ihrer fluviatilen und äolischen Umlagerungsprodukte (z. B. „toba"-Sedimente, HeINE \& Schönhals 1973) zusammengefaßt.

Vergesellschaftet mit den flächenmäßig dominierenden Gebieten, die von jüngeren Pyroklastika des Popocatépetl bedeckt sind, treten erodierte Bereiche mit geköptten Profilen $(5,15,16,17,18,19$ in Abb. 1) und Flächen mit fluviatil akkumulierten Mischsedimenten auf (20 in Abb. 1).

Die Verbreitung der beschriebenen Schichtabfolgen ist in ein Luftbildmosaik der Sierra Nevada de México eingetragen. Jede Kartiereinheit umfaßt mehrere Schichtabfolgen, die nach ihren Flächenanteilen in Leit- und Begleitprofile unterschieden werden.

Die Verbreitung der Aschenlagen 1C, 2C, 3C und c wurde bei der Kartierung aus ihrer stratigraphischen Stellung, vor allem im Zusammenhang mit den liegenden Bimslagen rekonstruiert. Falls der Schüttungsbereich der Aschen über das Areal der zugehörigen Bimslagen hinausging, liegen zwei oder mehrere Aschenlagen übereinander, die mit den bisher durchgeführten makro- und mikroskopischen Untersuchungen nicht unterschieden werden können. Weiterhin ist die Ansprache der 3C-Aschen nördlich des IztaccihuatlGipfels erschwert, da hier die liegende 3P-Bimslage nur noch vereinzelt auftritt. Es war daher nötig, die makroskopische Stratigraphie der insgesamt 54 für pedologische Untersuchungen entnommenen Profile mit laboranalytischen Untersuchungen zu überprüfen.

\section{Ergebnisse der Schwermineralanalyse}

Die untersuchten Profile verteilen sich angenähert gleichmäßig über ein Areal von ca. $70 \times 20 \mathrm{~km}$, v. a. nördlich des Haupteruptionsherdes Popocatépetl (Abb. 1). Alle in Abb. 1 angeführten Pyroklastika sind mindestens an 4, maximal an 61 Proben schwermineralytisch untersucht worden.

2) 14C-Datierung an einer Holzkohleprobe in Profil 3 der Abb. 1 durch das Niedersächsische Landesamt für Bodenforschung, Probenummer 4768 auf 990-1070 n. Chr. (dendrochronologisch korrigiert). 
Das luftrocken auf $<2 \mathrm{~mm}$ gesiebte Bodenmaterial wurde über $\mathrm{Nacht}$ in $0,04 \mathrm{n} \mathrm{NaOH}$ dispergiert (schwach basische Dispergierung wegen hoher Allophangehalte), 5 Minuten mit Ultraschall behandelt und naß auf $0,1 \mathrm{~mm}$ gesiebt. Der Rückstand wurde mit Tetrabromäthan $(\mathrm{d}=2,96)$ getrennt und die Fraktion $0,1-0,2 \mathrm{~mm}$ der Schwermineralfraktion trocken ausgesiebt.

Im Körnerpräparat (Einbettungsmittel Palatal, $\mathrm{n}=1,567$ ) wurden nach einer generellen Durchsicht des Präparats mindestens 300, max. 600 Körner ausgezählt. Die Auswahl der Körner erfolgte entlang paralleler Linien in gleichen Abständen.

Zur Fehlerabschätzung wurden $10 \%$ zufällig ausgewählte Proben doppelt analysiert. Der Variabilitätskoeffizient (berechnet für die Hyperstenkomponente) liegt bei $3 \%$ (Kornprozente). E.r beeinflußt also nur untergeordnet die in Abb. 2 bis 5 aufgetragenen Ergebnisse.

a). Aschenlage 1C

- Bimslage 1P b) Aschenlage 2C

- Bimslage 2P

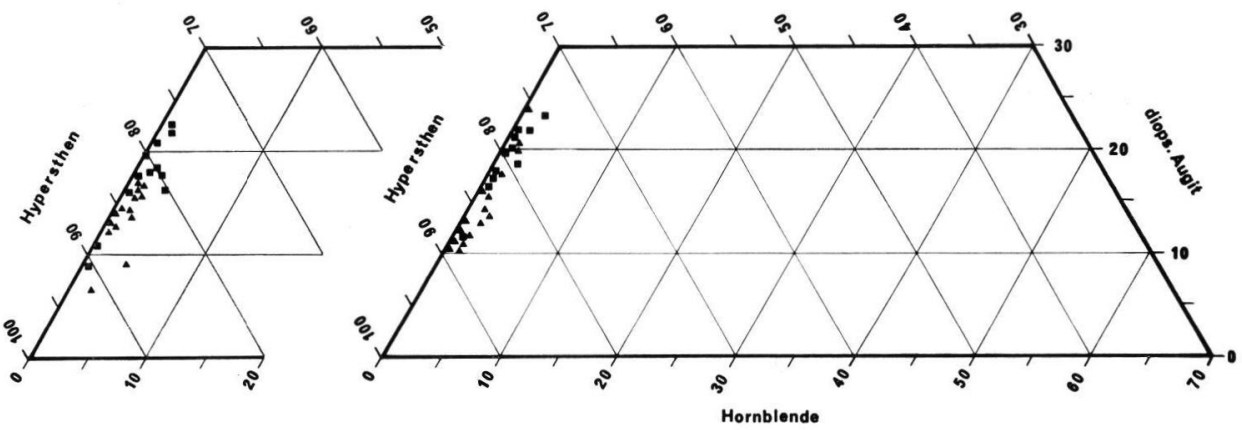

Abb. 2. Verhältnis von Hypersthen, diops. Augit und Hornblende der Aschen- und Bimslage 1C, 1P (a) und 2C, 2P (b) im Dreiecksdiagramm.

\section{Aschenlage 3C}

\section{Region}

A - - PopocatépetI

$\Delta-\cdots-\cdot$ Autopista

- -.-.- IztaccihuatI E

a...... Tlaloc/Telapon

- Iztaccihuat। W

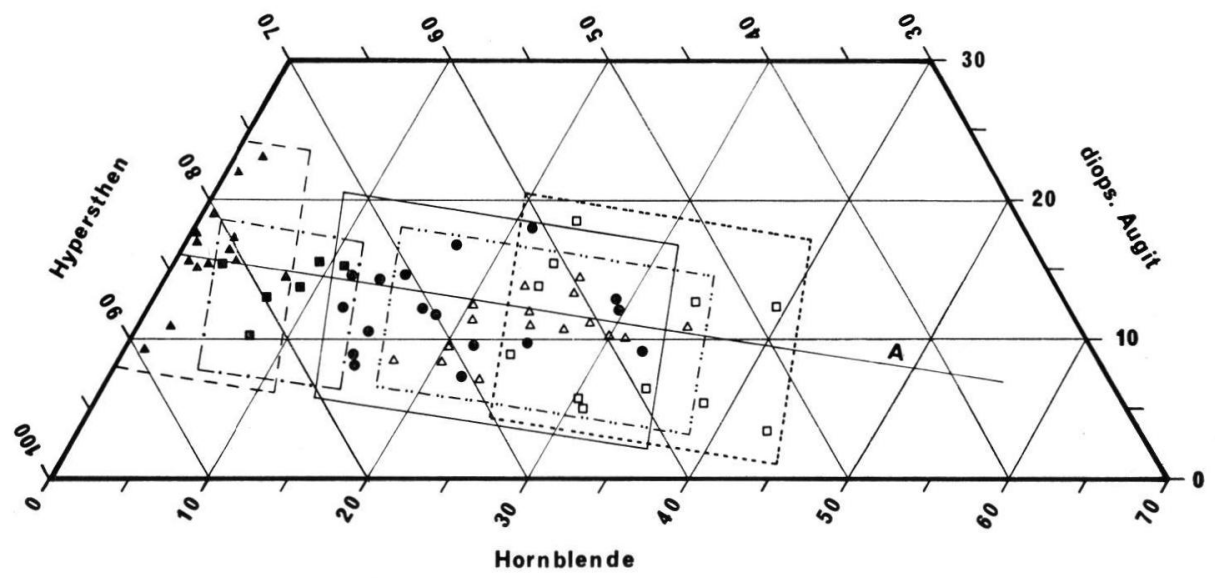

Abb. 3. Verhältnis von Hypersthen, diops. Augit und Hornblende der Aschenlage 3C im Dreiecksdiagramm. 
Die umfangreichen Schwermineralbestimmungen wurden von Frau Dr. H. HeideWEISE durchgeführt; die mineralogische Auswertung dieser Ergebnisse wird sie in einem gemeinsamen Artikel (MieHlich \& a. 1974) publizieren.

Die Schwermineralassoziation aller Proben ist sehr ähnlich. Hypersthen, diops. Augit und Hornblende machen durchschnittlich 99, maximal 100, minimal 90,5 Kornprozente der durchsichtigen Schwerminerale aus. Der Rest besteht aus Olivin. Da die geringen Olivingehalte keine Beziehungen zu den untersuchten Straten zeigen, die Unterschiede also ausschließlich in den relativen Verhältnissen der 3 Minerale Hypersthen, diops. Augit und Hornblende liegen, wurde die Summe dieser Minerale gleich $100 \%$ gesetzt. Auf diese Weise wird es möglich, die Beziehungen zwischen den drei verbleibenden Mineralen im Dreiecksdiagramm darzustellen. In Abb. 2 bis 5 wurde der Ausschnitt 70-100\% Hypersthen, 0-30\% diops. Augit, 0-70\% Hornblende aus dem Dreiecksdiagramm ausgewählt, da mit zwei Ausnahmen die Mineralverhältnisse aller Proben in dieser engen Begrenzung liegen.

Die 1C-Aschen- und 1P-Bimslage (Abb. 2) zeigen eine dichte Scharung der Werte zwischen 75-95\% Hypersthen, 5-25\% diops. Augit und nur 0-5\% Hornblende. Mit einer Begrenzung der 2C-Aschen- und 2P-Bimslage zwischen 75-90\%, Hypersthen, 10$25 \%$ diops. Augit und $0-5 \%$ Hornblende (Abb. 2) sind die Felder für 1C, 1P, 2C, $2 \mathrm{P}$ nahezu deckungsgleich. Sie lassen sich daher mit Hilfe der Schwermineralanalyse nicht auseinanderhalten. Die Bimslagen 1P und 2P tendieren zu höheren Gehalten an diops. Augit, die jedoch für eine Unterscheidung von den Aschenlagen nicht ausreichen.

Die Mineralverhältnisse der 3C-Aschenlage variieren stark. Sie umfassen den Bereich 45-90\% Hypersthen, 2,5-25\% diops. Augit und 0-45\% Hornblende (Abb. 3). Diese im Verhältnis zu 1C, 1P, 2C, 2P große Streuung der Werte wirft die Frage auf, ob bei der makromorphologischen Beschreibung Aschen unterschiedlicher Genese unter der Bezeichnung $3 \mathrm{C}$ zusammengefaßt wurden; eine Hypothese, die bei der relativen Inkonstanz der liegenden 3P-Lage denkbar ist. Die Gegenhypothese - alle Proben gehören einer Ausbruchsphase an - muß die starke Streuung der Schwermineralverhältnisse innerhalb eines Ausbruchs erklären.

Bei einer gewaltigen Eruption, die eine Aschenlage zwischen 1,8 und $1 \mathrm{~m}$ Mächtigkeit über eine Distanz von mindestens $70 \mathrm{~km}$ gefördert hat, sind vor allem zwei Mechanismen denkbar, die die Streuung der Mineralverhältnisse innerhalb einer Aschenlage erhöhen:

1. Eine Veränderung der Schwermineralzusammensetzung im Verlauf der Eruption, die sich in einer Änderung der Schwermineralverhältnisse innerhalb der untersuchten Profile ausdrücken müßte.

2. Eine Sortierung der Aschen, die sich in einer Veränderung der Schwermineralverhältnisse in Abhängigkeit von der Entfernung zum Ausbruchsherd zeigen müßte.

Beide Mechanismen lassen sich in den 3C-Aschen feststellen, wobei die Sortierung der Aschen überwiegt. Faßt man die Werte für 3C-Aschen regional zusammen (Abb. 3), so ergibt sich eine deutliche Abhängigkeit zur Entfernung vom Ausbruchsherd. Die Hornblendegehalte steigen zu Lasten der Gehalte an Hypersthen und diops. Augit von der Region Popocatépetl (Profile 2, 3, 4, 5, 7, 8, 9, Abb. 1) über die Regionen Iztaccihuatl E (Profile 22, 24), Iztaccihuatl W (Profile 13, 14, 15, 16, 17, 19) und Autopista (Profile 27, 29, 33, 35, 37, 41) zur Region Tlaloc/Telapón (Profile 44, 46, 48, 54) von ca. $2 \%$ auf über $30 \%$ stark an.

Die Streuung innerhalb der Regionen geht einerseits auf die Zusammenfassung unterschiedlich weit vom Ausbruchsherd entfernter Profile, andererseits auf die Unterschiede innerhalb der Profile (vgl. unten) zurück. Die Varianz innerhalb der Regionen steigt 
mit der Entfernung zum Ausbruchsherd, da die Konstanz der Ablagerungsbedingungen mit zunehmender Entfernung abnimmt.

Noch deutlicher wird die angenähert linear entfernungsabhängige Veränderung der Schwermineralverhältnisse der 3C-Aschen, wenn man die Hornblendegehalte zur Entfernung zum Krater in Beziehung setzt. (Tab. 1, die beiden ersten Ziffern jeder dreistelligen Zahl geben die Profilnummer der Probenahme an - vgl. Abb. 1 -, die dritte Ziffer ist die Probennummer innerhalb des Profils, Probe $10=\overline{0}$ ).

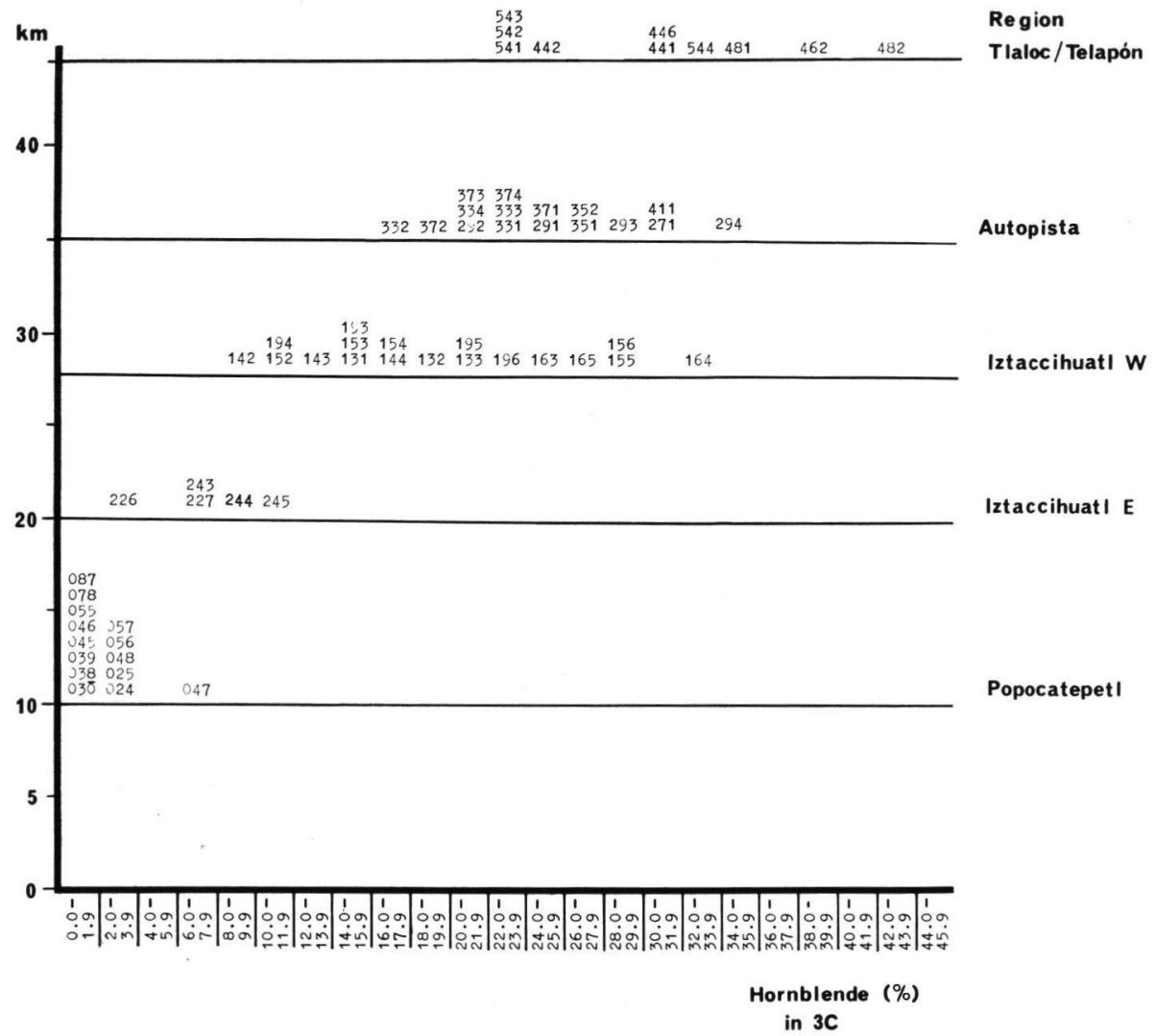

'Tab. 1. Beziehung zwischen dem Hornblendegehalt in 3C-Aschen und der Entfernung zum Krater des Popocatépetl (Kornprozent der Schwerminerale der Fraktion 0,1-0,2 mm).

Die Gerade A in Abb. 3 geht von einer Mineralzusammensetzung 84\% Hypersthen, $16 \%$ diops. Augit und $0 \%$ Hornblende aus und verläuft nach $0 \%$ Hypersthen und diops. Augit und 100\% Hornblende; sie stellt also die relative Veränderung von Hypersthen und diops. Augit bei steigenden Hornblendegehalten dar. Die enge Scharung der Werte um diese Ausgleichsgerade zeigt, daß es sich bei der Varianz der Mineralzusammensetzung innerhalb der 3C-Aschen lediglich um eine relative Anreicherung von Hornblende handelt.

Alle diese Ergebnisse deuten auf eine starke Sortierung der 3C-Aschen bei ihrer Ablagerung. Als Ursache für eine Sortierung vulkanischer Feinaschen kommen Unterschiede in der Korngröße, in der Kornform und im spez. Gewicht der untersuchten Minerale in Frage. Körnungsunterschiede können bei der relativ engen Fraktion von 0,1-0,2 mm 
ebenso wie die Kornform nur eine untergeordnete Rolle spielen. Es ist daher der verhältnismäßig geringe Unterschied im spezifischen Gewicht $\left(3,5-3,7 \mathrm{~g} / \mathrm{cm}^{3}\right.$ für Hypersthen, 3,3-3,5 für Augit und 2,9-3,4 für Hornblende, nach Kaltofen \& a. 1972) als Hauptursache für die gleichsinnige Abnahme von Hypersthen und diops. Augit bei steigenden Hornblendegehalten anzusehen.

In der Literatur (FRECHEN 1952; WeYl 1954) wird gerade das Hornblende : AugitVerhältnis wegen des ähnlichen spez. Gewichts als ein auch über große Entfernungen vom Eruptionsherd konstantes Merkmal vulkanischer Lockerprodukte angesehen. In beiden Fällen wurden Bimslagen untersucht, bei denen die Schwerminerale in einem Gerüst aus Glas eingebettet sind. Die Flugeigenschaften der Bimspartikel werden dabei unwesentlich vom spez. Gewicht der meist nur wenige Gewichtsprozent betragenden Schwerminerale beeinflußt. In den hier untersuchten Feinaschen wurden, nach dem Zustand der Minerale zu urteilen, die Schwerminerale vor allem als Einzelkristalle transportiert, so daß sich die unterschiedlichen Flugeigenschaften der Schwerminerale selbst voll auswirken. Unter diesen Bedingungen können sich Schwermineralverhältnisse offensichtlich bereits über sehr kurze Distanzen stark ändern.

Allgemein gültige Entfernungsregeln lassen sich jedoch nicht ableiten. Nimmt man an, daß die Linien gleicher Sortierung, ähnlich wie Isopachen, angenähert elliptisch um das Ausbruchszentrum angeordnet sind (MEYER 1964), so steigt die Sortierung in Abhängigkeit von der Entfernung - je nach Richtung zum Krater - unterschiedlich stark. Für die hier untersuchten 3C-Aschen des Popocatépetl ist das Förderareal nicht bekannt, es kann daher auch die Lage der untersuchten Profile zum Gesamtareal nicht bestimmt werden.

Neben dieser entfernungsabhängigen Veränderung läßt sich auch eine Verschiebung der Schwermineralzusammensetzung im Verlauf der Eruption der 3C-Aschen feststellen. Der Hornblendegehalt sinkt bei vielen Profilen innerhalb der 3C-Aschen von unten nach oben deutlich ab, wobei auch hier die relative Abnahme bei zunehmender Entfernung der Profile vom Ausbruchsherd größer wird; ein weiterer Hinweis auf die gleichmäßige Schüttung der 3C-Aschen über den gesamten untersuchten Bereich. Der Kurvenverlauf entspricht der Veränderung des Magmas zu einer stärker basischen Zusammensetzung bei abnehmendem Gasdruck während dieser Eruption (frdl. mündl. Mittlg. Dr. HeIdE-WeISE).

Als Unterscheidungsmerkmal läßt sich die Schwermineralzusammensetzung der 3CAschen nicht verwenden. Im Bereich des Popocatépetl gleichen sich - bei geringen Hornblendedehalten - die Schwermineralverhältnisse von 1C, 2C und 3C, während weiter nördlich - bei steigenden Hornblendegehalten - die älteren Pyroklastika $c$ und die vulkanischen Sande A von 3C nicht unterschieden werden können.

Die Schwermineralzusammensetzung der 3P-Bimslagen liegt im Diagrammfeld 35$65 \%$ Hypersthen, 10-30\% diops. Augit und 20-45\% Hornblende. Sie zeigt im Gegensatz zu den 3C-Aschen keinen Zusammenhang zur Entfernung vom Ausbruchsherd. Bei der heterogenen Zusammensetzung und bei Korngrößen in der Kiesfraktion ist eine Sortierung, wie sie für die 3C-Aschen beobachtet wurde, auch nicht zu erwarten.

Die Schwermineralverhältnisse der älteren Aschen c streuen, wie die 3C-Aschen, in einem weiten Bereich zwischen 15-90\% Hypersthen, 0-20\% diops. Augit und 0-75\% Hornblende (Abb. 4). Auch hier deuten die enge Scharung der Werte um eine der Ausgleichsgeraden $\mathrm{A}$ in Abb. 3 ähnelnden Linie und die Beziehung der Hornblendegehalte zur Entfernung vom Popocatépetl auf Ablagerungsbedingungen wie sie für die 3C-Asche beschrieben wurden.

Die Wahrscheinlichkeit einer Scheinkorrelation ist jedoch ziemlich hoch, da das mächtige, vielfältig zusammengesetzte Paket der c-Aschen stratigraphisch durchgehend nicht untergliedert werden konnte. Es kann daher nicht entschieden werden, ob die unter der 
Bezeichnung c zusammengefaßten Pyroklastika einem Ausbruchzentrum entstammen und welche Lage, erosiv bedingt, heute oberflächlich ansteht und damit bei der Probenahme erfaßt wurde. Darüber hinaus zeigen die Substrate c teilweise Anzeichen von Umlagerungen durch Wasser- und Windtransport (Heine \& Schönhals 1973).

Ältere Pyroklastika (c)

Profile

- 10

- $13,14,15$

$\triangle 26$

$\times 27$

- 24,25

- $27,41,42,44,46$

- 43

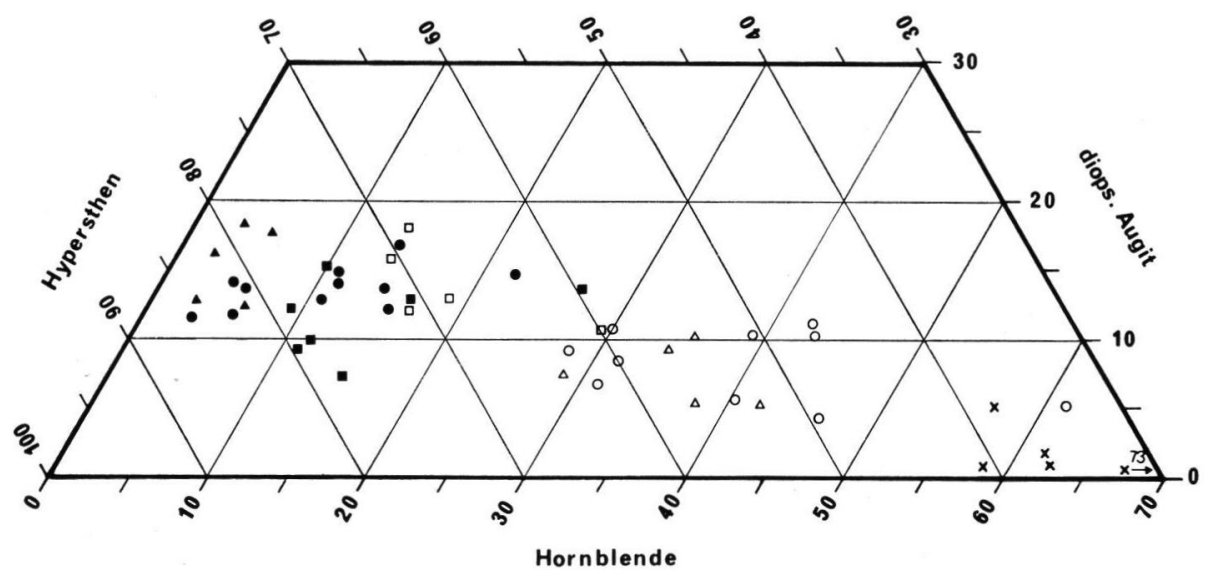

Abb. 4. Verhältnis von Hypersthen, diops. Augit und Hornblende der älteren Pyroklastika c im Dreiecksdiagramm.

- Sedimente fluviatil akkumuliert

- Gesteinszersatz

- glazial aufbereitetes Sediment

$\triangle$ vulkanische Sande Tlaloc/Telapón

- 3C Aschen und vulkanische Sande Tlaloc/Telapón

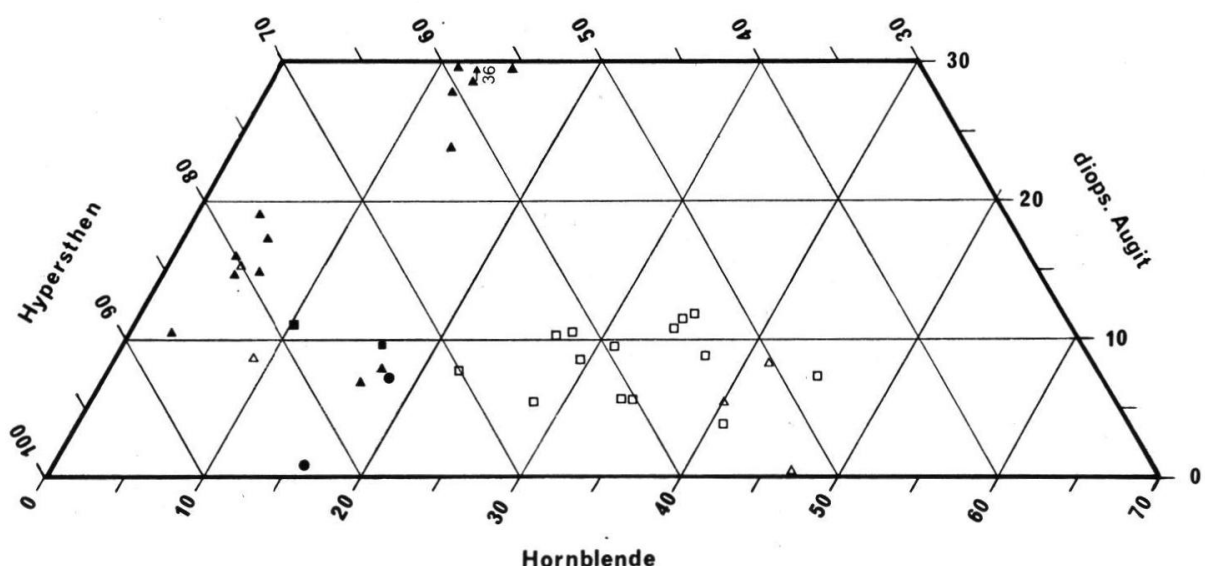

Abb. 5. Verhältnis von Hypersthen, diops. Augit und Hornblende im Dreiecksdiagramm. 
Von den fluviatil transportierten Sedimenten (S) wurden zwei Beispiele ausgewählt (Profil 1 und 18, Abb. 5), die einerseits zeigen, daß die Schwermineralzusammensetzung dieser Sedimente je nach Einzugsgebiet verschieden ist (Hornblendegehalte) und daß andererseits eine starke Streuung innerhalb eines Profils auftreten kann (Profil 18).

Die makroskopisch leicht $\mathrm{zu}$ unterscheidenden 3C-Aschen und vulkanischen Sande haben im Tlaloc/Telapón-Gebiet sehr ähnliche Schwermineralzusammensetzungen. Die Differenzierung der makromorphologisch nur schwer unterscheidbaren Mischformen ist daher mit Hilfe der Schwermineralanalyse nicht möglich (Abb. 5).

Zusammenfassend läßt sich sagen, daß bei der sehr ähnlichen Schwermineralzusammensetzung aller bodenbildenden Substrate und der starken Windsichtung von Aschen großer Reichweite, die Schwermineralanalyse keine geeignete Methode zur Unterscheidung der jüngeren Pyroklastika der Sierra Nevada de México ist. Es wurde daher versucht, eine Stratigraphie mit pedologischen Untersuchungen durchzuführen.

\section{Pedologische Untersuchungen}

Bei der Verwendung schwermineralanalytischer Ergebnisse zur Stratigraphie der Pyroklastika wird davon ausgegangen, daß sich die untersuchten Eigenschaften der vulkanischen Lockerprodukte seit ihrer Ablagerung nicht verändert haben und zum Zeitpunkt der Untersuchung die ursprünglichen Eigenschaften der Straten widerspiegeln. Im Gegensatz dazu wird bei der Verwendung von Bodeneigenschaften die altersabhängige Veränderung der Eruptiva zum Kriterium der Stratigraphie.

3.1. Voraussetzungen für die Anwendbarkeit pedologischer Untersuchungen zur Stratigraphie von Pyroklastika

Unter der großen Zahl von Bodeneigenschaften eignen sich nur wenige Merkmale zur Stratigraphie, da sie mehrere Voraussetzungen erfüllen müssen.

\subsubsection{Monotone Altersfunktion}

Oberflächennahe Pyroklastika werden unter dem Einfluß von Klima, Organismen, Relief und Mensch zu Böden umgestaltet. Dabei verändern sich die Bodeneigenschaften, die ihrerseits als Fließgleichgewichte aufgefaßt werden können, mit der Zeit. Zur Strati-

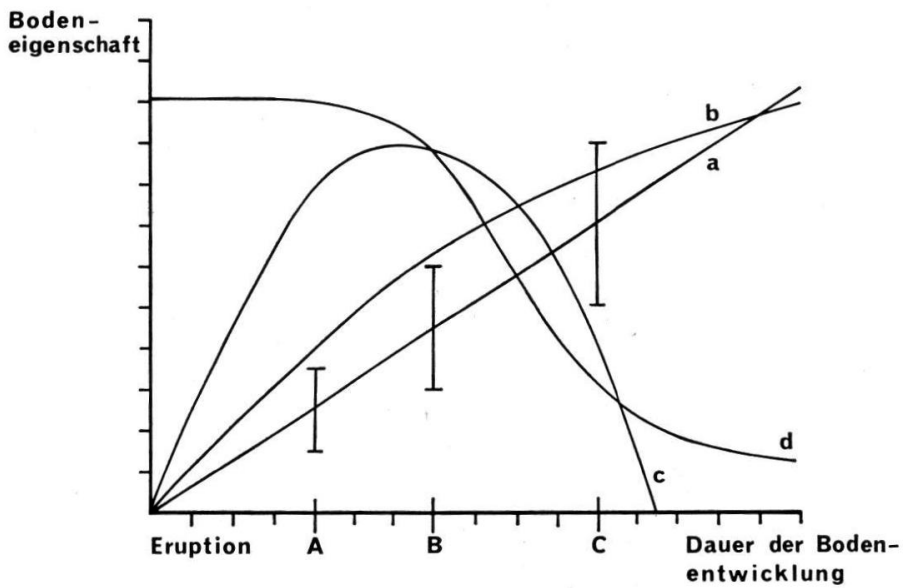

Abb. 6. Schema möglicher Beziehungen zwischen Bodeneigenschaften und Dauer der Bodenentwicklung; weitere Erläuterungen im Text. 
graphie müssen Bodeneigenschaften herangezogen werden, deren zeitabhängige Veränderung über den betrachteten Zeitraum keine Maxima oder Minima aufweist, da sonst Pyroklastika unterschiedlichen Alters gleiche Werte der Testgröße annehmen können (Kurve c in Abb. 6). So wird z. B. der Allophangehalt in Andosolen, der altersabhängig zunächst zunimmt, schließlich aber durch Kristallisation zu Tonmineralen wieder abnimmt, keine geeignete Testgröße zur Unterscheidung der Eruptiva der Ausbrüche A, B und C in Abb. 6 sein. Er kann dagegen benutzt werden, um noch ältere Aschenlagen, die kein Allophan mehr enthalten, von jüngeren Aschenlagen mit Allophan zu unterscheiden. Ideal sind Eigenschaften, die sich bei möglichst geringer Beeinflussung durch die übrigen Faktoren linear zur Dauer der Bodenentwicklung verändern, da unter diesen Voraussetzungen neben einer relativen Datierung auch Schätzungen des absoluten Alters möglich sind (Kurve a in Abb. 6). Häufig werden es jedoch asymptotische Kurven vom Typ b sein, die lediglich eine relative Einordnung möglich machen.

\subsubsection{Geringe Tiefenfunktion}

Geeignete Testgrößen sind häufig Produkte von Transformationsprozessen, die meist tiefenabhängige Veränderung der Werte zeigen. Die hieraus resultierende Streuung innerhalb einer Schicht hängt vor allem vom Boden- und Klimatyp ab. Im allgemeinen nehmen die Werte mit zunehmender Tiefe ab. Bei starkem Tiefengradient muß das gewogene Mittel der Schicht gebildet werden. Wenn bei stark tiefenabhängiger Veränderung in Hanglagen der Oberboden durch Erosion abgetragen wird, verlagert sich die Bodenbildung in ehemals tieferliegendes, wenig durch Bodenbildung verändertes Material. Diese scheinbare Verjüngung des Bodens erschwert die Stratigraphie mit pedologischen Mitteln.

Ein Sonderfall tiefenabhängender Bodenentwicklung tritt in Vulkangebieten auf, in denen die Förderprodukte älterer Eruptionen bei jüngeren Ausbrüchen nur teilweise bedeckt wurden. Die Bodenentwicklung der älteren Schicht wird im Gebiet ohne Überdekkung intensiver ablaufen als im überdeckten Areal. Die Überdeckung bedeutet in jedem Fall eine Veränderung der Entwicklungsintensität; wird sie bei tiefgründiger Überdeckung nahezu Null, so wird der Boden in seinem Stadium zum Zeitpunkt der nächstjüngeren Eruption konserviert. Es kann jedoch auch zu einer Rückentwicklung einzelner Bodenmerkmale kommen (z. B. Humusgehalt).

\subsubsection{Verlagerungsresistenz}

$\mathrm{Da}$ in Böden neben Transformationsprozessen, deren Auswirkungen wir zur Stratigraphie heranziehen, auch Translokationsprozesse ablaufen, muß überprüft werden, inwieweit das untersuchte Merkmal verlagerungsresistent ist. Das Ausmaß der Verlagerungsvorgänge ist vor allem typgebunden. So ist z. B. in jungen Andosolen die infiltrative Beweglichkeit des Humus sehr groß, während die Verlagerung pedogener anorganischer Verbindungen wie Allophan und Eisenoxide sehr gering ist. Altern diese Böden, so zeigen sie häufig Merkmale einer Lessivierung, ein Prozeß, der durch hohe Beweglichkeit der Tonminerale und Eisenoxide charakterisiert ist.

Solange die Verlagerung auf die zu untersuchende Schicht beschränkt ist, wird lediglich die Streuung der Vergleichswerte innerhalb der Schicht erhöht. Dies ist unbedenklich, solange die altersabhängigen Unterschiede deutlich größer sind. Bei starker Streuung muß ebenfalls das gewogene Mittel einer Schicht gebildet werden. Die Testgröße wird unbrauchbar, wenn die Verlagerung auf tieferliegende Schichten übergreift.

\subsubsection{Isolierbarkeit der altersabhängigen Bodenentwicklung}

In Abbildung 6 wurde die altersabhängige Veränderung einer Bodeneigenschaft schematisiert als Linie dargestellt. Diese Schärfe der Beziehung gilt jedoch nur unter der 
Voraussetzung, daß die übrigen Faktoren der Bodenbildung im gesamten Untersuchungsgebiet und über den betrachteten Zeitraum konstant waren, eine Prämisse, die unter natürlichen Bedingungen nicht erfüllt ist.

Die Zeitfunktion der Bodenentwicklung wird daher für die verschiedenen innerhalb des Untersuchungsgebietes auftretenden Faktorenkonstellationen variieren. In Abb. 6 ist diese Streuung durch vertikale Balken um die Funktion „a “ für drei verschieden alte Pyroklastika (A, B, C) dargestellt. Sie umfaßt außerdem die aus dem Analysenfehler, der Tiefenfunktion und aus Verlagerungsvorgängen resultierende Streuung. Eine sichere Zuordnung der untersuchten Pyroklastika ist mit Hilfe der Bodeneigenschaft nur möglich, wenn sich die Streubereiche der einzelnen Eruptionen nicht überschneiden. In Abb. 6 lassen sich die Böden aus Pyroklastika der Eruptionsphasen A und C eindeutig unterscheiden, während für $\mathrm{A} / \mathrm{B}$ und $\mathrm{B} / \mathrm{C}$ Uberschneidungsbereiche bestehen, in denen eine Zuordnung nicht möglich ist. Die Abbildung zeigt weiter, daß die Unterscheidbarkeit verschieden alter Pyroklastika mit Hilfe eines Bodenmerkmals von den Relationen Altersunterschied der Pyroklastika zu Steigerung der Altersfunktion des Bodenmerkmals zu Varianz des Merkmals gleichalter Pyroklastika abhängt.

Voraussetzung für diese Art der Schlußfolgerung ist, daß zumindest die Extremwerte der variierenden Bodenbildungsfaktoren erfaßt werden, damit die maximale Spannweite der Streuung bekannt ist. Eine streng statistische Probenahme (z. B. geschichtete Stichprobe) wird bei den räumlich gleitenden Übergängen der unterschiedlichen Faktorenkonstellationen und dem vom Arbeitsaufwand her bestimmten kleinen Stichprobenumfang nur selten möglich sein.

Für die einzelnen Faktoren muß insbesondere folgendes berücksichtigt werden:

Mineralzusammensetzung und Form der Pyroklastika müssen sehr ähnlich sein. Auch bei vergleichbarem Chemismus einer Bims- und einer Aschenlage wird die altersabhängige Bodenentwicklung sehr unterschiedlich ausfallen, da sich vor allem der Wasserhaushalt in beiden Substraten erheblich unterscheidet.

$\mathrm{K} l$ im a varianzen innerhalb des Untersuchungsgebiets beeinflussen die altersabhängige Bodenentwicklung und erhöhen so die Streuung der Testgröße innerhalb einer Schicht. Der Einfluß der Klimaunterschiede steigt mit dem Alter der untersuchten Schichten. Lassen die auftretenden Klimavarianzen einen nennenswerten Einfluß auf die Testgröße vermuten, so müssen bei der Probenahme die klimatischen Extremlagen berücksichtigt werden, um die Spannweite der hieraus resultierenden Streuung zu erfassen. Ebenso sind die Auswirkungen paläoklimatischer Klimaschwankungen zu überprüfen.

Reli ef unterschiede bedingen vor allem eine Änderung der Erosionsanfälligkeit und des Wasserhaushalts. Die übrigen Faktoren wirken nur in Einzelfällen stärker differenzierend.

\subsection{Eignung pedogener Eisenfraktionen zur stratigraphischen Gliederung der jüngeren} Pyroklastika der Sierra Nevada de México

Untersuchungen zur alters- und klimaabhängigen Bodenentwicklung der Sierra Nevada (MieHLICH 1974) haben gezeigt, daß Eisenfraktionen als pedologische Testgröße zur Unterscheidung der makroskopisch, mikroskopisch und schwermineralanalytisch nicht unterscheidbaren Aschenlagen 1C, 2C, 3C und c geeignet sein können. Bestimmt wurden an insgesamt 157 Proben Gesamteisen $\left(\mathrm{Fe}_{\mathrm{t}}\right)$ und an 237 Proben dithionitlösliches Eisen $\left(\mathrm{Fe}_{\mathrm{d}}\right)$ und oxalatlösliches Eisen $\left(\mathrm{Fe}_{0}\right)$. Das Gesamteisen wurde im $\mathrm{HF} / \mathrm{HClO}_{4}$-Aufschluß (JACKSON 1958 modifiziert) erfaßt, die Extraktion mit $\mathrm{Na}_{2} \mathrm{~S}_{2} \mathrm{O}_{4}$ erfolgte nach MEHrA \& JACKsON (1960) und das oxalatlösliche Eisen wurde nach SCHWERTMANN (1964) gewonnen. In allen Fraktionen wurde das Eisen atomabsorptionsspektrometrisch bestimmt und auf $\mathbf{0} \%$ der 
humusfreien Feinerde berechnet. Der Quotient $\mathrm{Fe}_{\mathrm{o}}: \mathrm{Fe}_{\mathrm{d}}$ wird als Aktivitätsgrad bezeichnet.

Durch die Verwitterung werden aus dem Substrat im Verlauf der Bodenbildung FeIonen freigesetzt, die teils als röntgenamorphe Eisenoxidhydrate ausfallen, teils organische Komplexverbindungen eingehen. In Abhängigkeit von Bodeneigenschaften kristallisiert ein wechselnder Anteil zu sekundären Eisenmineralen. Das dithionitlösliche Eisen ist ein Maß für den Gesamtgehalt an pedogenem Eisen, während das oxalatlösliche Eisen lediglich ein Maß für das gelöste, organisch gebundene und röntgenamorphe Eisen ist. Der Quotient $\mathrm{Fe}_{\mathrm{o}}: \mathrm{Fe}_{\mathrm{d}}$ ist daher ein Indikator für den Kristallisationsgrad des pedogenen Eisens. Die Anwendbarkeit dieser Fraktionierung zur Interpretation bodengenetischer Prozesse in mitteleuropäischen Böden untersuchten Blume \& SChwertmann (1969); spezielle Probleme bei Pyroklastika diskutieren Meyer \& SAKR (1970).

$\mathrm{Da}$ Na-Dithionit auch das in Pyroklastika auftretende primäre Mineral Magnetit löst, ist die Methode nur bei magnetitarmen Substraten einsetzbar.

Es soll nun im einzelnen überprüft werden, inwieweit die pedogenen Eisenfraktionen die Voraussetzungen von Abschnitt 3.1 erfüllen.

Monotone Altersfunktion: der Gehalt an Fed wird über den hier betrachteten Zeitraum bei einer hohen Reserve an verwitterbaren Mineralen altersabhängig zunehmen; ein linear zeitabhängiger Zusammenhang ist u. a. wegen der unterschiedlichen Verwitterbarkeit von Gläsern und Mineralen nicht zu erwarten (Kurventyp b in Abb. 6). Der Aktivitätsgrad $\mathrm{Fe}_{\mathrm{o}}: \mathrm{Fe}_{\mathrm{d}}$ wird während der Andosolphase zunächst altersunabhängig hoch sein; erst gegen Ende der Allophanphase wird der Aktivitätsgrad altersabhängig abnehmen, da bei sinkenden Allophan- und Humusgehalten die Kristallisationshemmung zu sekundären Eisenmineralen geringer wird (Kurventyp d in Abb. 6).

Geringe Tiefenfunktion : der Tiefengradient der Eisenfraktionen ist im Bereich der Sierra Nevada entsprechend ihrer Lage im Klimabereich der Randtropen gering. Die Substratkarte (Abb. 1) zeigt, daß sich die Areale für $1 \mathrm{C} / 1 \mathrm{P}, 2 \mathrm{C} / 2 \mathrm{P}$ und $3 \mathrm{C} / 3 \mathrm{P}$ nicht decken. Es war daher notwendig, die Proben der $3 \mathrm{C}$-Aschen in $3 \mathrm{C}_{1}$ (unbedeckt) und $3 \mathrm{C}_{2}$ (von $1 \mathrm{C}-2 \mathrm{P}$ bedeckt) getrennt zu betrachten. Die Aschen $2 \mathrm{C}$ treten in den untersuchten Proben immer von $1 \mathrm{C}$ und $1 \mathrm{P}$ bedeckt auf.

Verlagerungsresistenz: eine Verlagerung von pedogenem Eisen ist für die Aschen $1 \mathrm{C}$ und $2 \mathrm{C}$ auszuschließen, während die Tiefenfunktionen des $\mathrm{Fe}_{\mathrm{d}}-\mathrm{Gehalts} \mathrm{der}$ 3C-Profile im warm trockenen Bereich des Hangfußes der Sierra Nevada eine schwache Verlagerung andeuten, die jedoch die Schichtgrenzen nicht überschreitet. Eine relative Anreicherung des pedogenen Eisens durch Abfuhr anderer anorganischer Komponenten (z. B. Desilifizierung vgl. Aeppli 1973) kann für die jungen Aschenlagen 1C bis 3C wegen der geringen Tiefenfunktion des natronlaugelöslichen Siliciums (MieHLich 1974) nur untergeordnet wirksam sein.

Isolierbarkeit der altersabhängigen Bodenentwicklung: die einheitliche Schwermineralparagenese der Aschen 1C, $2 \mathrm{C}$ und die übereinstimmenden Gesamteisengehalte $(1 \mathrm{C}: 36-46 \%$; $2 \mathrm{C}: 38-50 \%$ ) zeigen, daß für $1 \mathrm{C}$ und $2 \mathrm{C}$ die primäre Einheitlichkeit sehr hoch ist. Die starke Windsortierung der 3C-Aschen erhöht die Inhomogenität der primären Zusammensetzung, was sich in einer höheren Streuung der $\mathrm{Fe}_{\mathrm{t}}$-Werte $\left(36-56 \%\right.$ ) ausdrückt. Es ist daher zu erwarten, daß die Streuung der $\mathrm{Fe}_{\mathrm{d}^{-}}$ Werte innerhalb $3 \mathrm{C}$ durch die Inhomogenität des Ausgangsmaterials erhöht wird. Beim Vergleich zwischen den Schichten 1C, 2C und 3C ist ein geringes Ansteigen der $\mathrm{Fe}_{\mathrm{t}}$-Gehalte mit zunehmendem Alter zu berücksichtigen, was eine leichte Überzeichnung des Alterseinflusses auf $\mathrm{Fe}_{\mathrm{d}}$ bewirken könnte. $\mathrm{Da}$ die Streubreite der $\mathrm{Fe}_{\mathrm{t}}$-Werte für alle Schichten jedoch überdeckend ist, kann dieser Faktor nur untergeordnet wirksam sein. 
Sowohl die Ergebnisse der Schwermineralanalyse als auch die hohe Streubreite der $\mathrm{Fe}_{\mathrm{t}^{-}}$ Werte $(35-75 \%$ oo $)$ zeigen, daß unter dem Oberbegriff „ältere Pyroklastika“ so heterogene Substrate zusammengefaßt wurden, daß im direkten Vergleich die $\mathrm{Fe}_{\mathrm{d}}$-Werte weder innerhalb von $\mathrm{c}$ noch gemeinsam mit $1 \mathrm{C}, 2 \mathrm{C}$ und $3 \mathrm{C}$ zur Altersgliederung benutzt werden können. Dies gilt in verstärktem Maße für die Substrate S, G, M und A. Die Bimslagen 1P, 2P und 3P können trotz ähnlicher Schwermineralparagenese und Gesamteisenwerte wegen der unterschiedlichen Bodenentwicklung nicht mit den Aschen 1C, 2C und 3C verglichen werden.

Untersuchungen von LAUeR \& STIEHL (1973) ergaben für das Arbeitsgebiet eine beträchtliche höhenabhängige Klimavarianz, die einen starken Einfluß auf die Eisenfreisetzung hat (Mienlich 1974). Es ist daher eine von 1C über 2C zu 3C zunehmende Streuung der $\mathrm{Fe}_{\mathrm{d}}$-Gehalte zu erwarten. Die Probenahme berücksichtigt für alle Pyroklastika die maximale Klimavarianz. Nach den paläoklimatischen Untersuchungen von HeINE (1973) gab es in der Sierra Nevada im betrachteten Zeitraum vier Phasen mit kühler-feuchteren Klimabedingungen als heute, die zu Gletschervorstößen führten. Auch aus diesem Grunde ist mit einer linear altersabhängigen Zunahme der $\mathrm{Fe}_{\mathrm{d}}$-Gehalte nicht $\mathrm{zu}$ rechnen.

Die übrigen Faktoren der Bodenbildung (Vegetation, Relief und wirtschaftender Mensch) sind in der Sierra Nevada im Verhältnis zur altersabhängigen Bodenentwicklung nur untergeordnet wirksam. Dies ist besonders für das Relief erstaunlich, da Hangneigungen bis $70 \%$ keine Seltenheit sind. Die splittrigen, ineinander verzahnten Einzelkörner bedingen bei hoher Wasserleitfähtigkeit eine starke Resistenz gegen Erosion, solange die geschlossene Vegetationsdecke erhalten bleibt. Weganschnitte, die Bachbetten queren, zeigen, daß die Erosionsleistung seit den letzten beiden Eruptionen sehr gering war. Die Gefahr flächenhafter Erosion birgt die Vernichtung der Krautschicht und der organischen Auflage durch häufiges Brennen in Waldweidegebieten (ERN \& MieHLICH 1972).

\subsection{Ergebnisse der Eisenfraktionierung und des Allophan-Tests}

In Tab. 2 bis 5 sind die Gehalte an dithionitlöslichem Eisen bzw. die Quotienten $\mathrm{Fe}_{\mathrm{o}}: \mathrm{Fe}_{\mathrm{d}}$ der untersuchten Substrate als Häufigkeitsverteilung aufgetragen (vgl. Erläuterungen zu Tab. 1 in Abschnitt 2). Für die Pyroklastika 1C, 1P, 2C, 2P, 3C, 3P und c umfaßt die Probenahme alle Regionen und alle Extreme des am stärksten differenzierenden Faktors Klima. Es ist daher anzunehmen, daß die Spannweite der Verteilung für diese Pyroklastika charakteristisch ist. Dagegen hat die Form der ermittelten Verteilungen wegen der systematischen Probenahme bei teilweise geringem Stichprobenumfang wenig Aussagewert. Für die Lagen A, S, G und M wurden jeweils nur einzelne charakteristische Beispiele ausgewählt.

Der Analysenfehler (bestimmt an 10\% zufällig ausgewählter Proben) ist mit einem Variabilitätskoeffizient unter $2 \%$ so gering, daß die Streuung durch den Analysenfehler nur unwesentlich erhöht wird.

Proben, deren Zuordnung makromorphologisch nicht eindeutig möglich war, werden getrennt aufgeführt $(1 \mathrm{C} / 2 \mathrm{C}, 2 \mathrm{C} / 3 \mathrm{C}, 3 \mathrm{C} / \mathrm{c}, 3 \mathrm{C} / \mathrm{A})$. Solche Proben treten vor allem in Profilen auf, die entlang der Verbreitungsgrenzen der Bimslagen entnommen wurden (Tab. 2 u. 4, Profile 02, 14, 15, 16, 19, 24), da hier teilweise die trennende Bimslage fehlt, und so die makromorphologisch nicht unterscheidbaren Aschenlagen direkt übereinanderliegen. Die Proben 3C/c, 3C/A treten entweder in Profilen auf, in denen die 3P-Lage fehlt oder undeutlich ausgebildet ist bzw. Durchmischungen beider Substrate vorliegen (Tab. 3 u. 5).

Die $\mathrm{Fe}_{\mathrm{d}}$-Gehalte der jüngsten Aschenlage 1C (Tab. 2) sind mit 1,0-4,9 \%/oo sehr gering; ihre Streuung ist trotz hoher Klimavarianz entsprechend des geringen Alters von $1 \mathrm{C}$ 


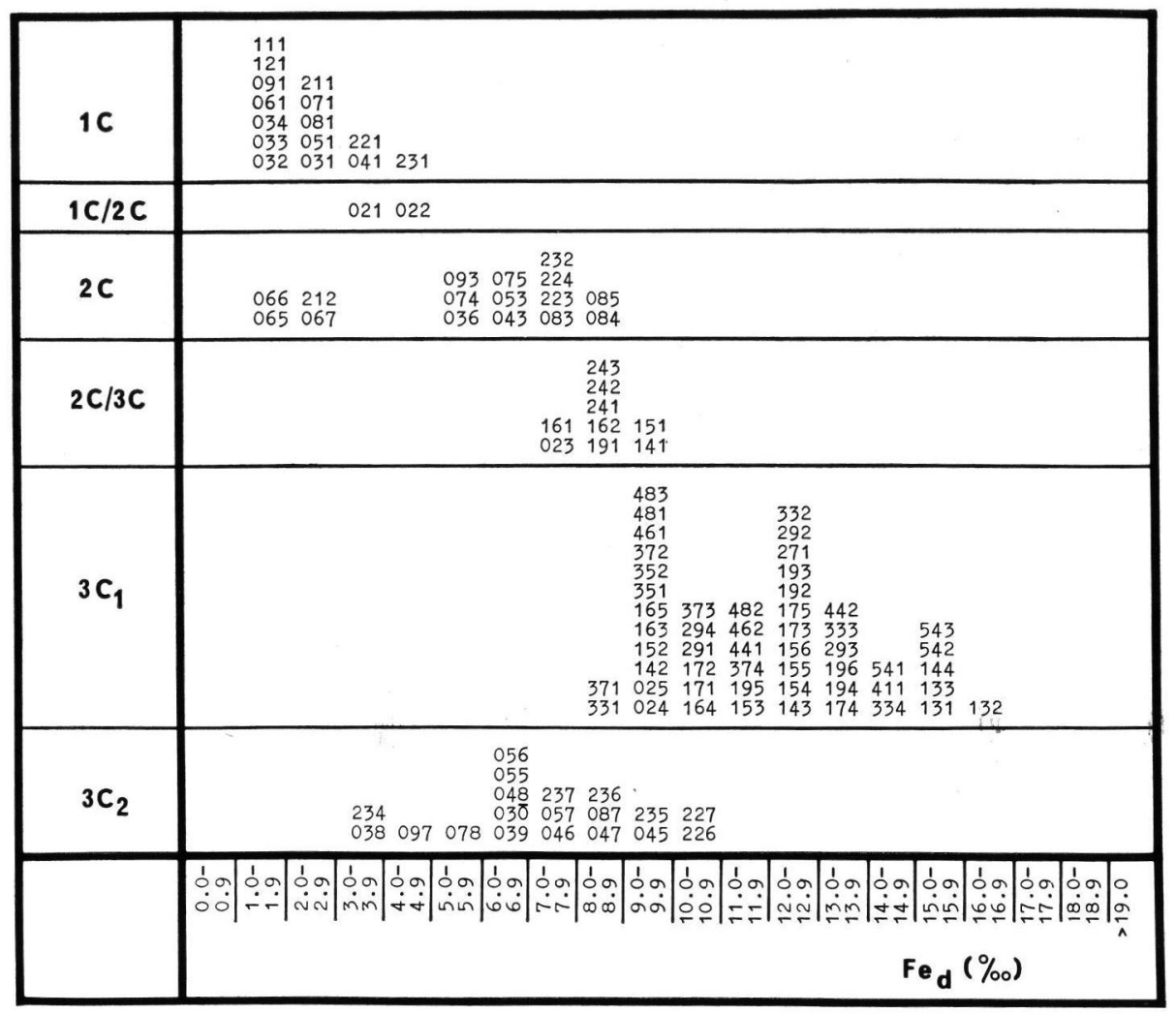

Tab. 2. Häufigkeitsverteilung des Gehalts an dithionitlöslichem Eisen $\left(\mathrm{Fe}_{\mathrm{d}}\right)$ in $\%$ oo der humusfreien Feinerde; Abkürzungen vgl. Abschnitt 1.

(ca. 400 Jahre) nur wenig ausgeprägt. Der hohe Wert der Probe 231 ist möglicherweise auf eine Beimengung von 2C-Aschen zurückzuführen, da in Profil 23 die 1P-Lage nur als dünner Schleier ausgebildet ist.

Die $\mathrm{Fe}_{\mathrm{d}}$-Werte für die 2C-Aschen bilden zwei Verteilungen. Die geringen Gehalte zwischen 1,0 und 2,9\% entstammen Profilen in mehr als $4000 \mathrm{~m}$ Meereshöhe. Unter den Klimabedingungen dieser Region (mittlere Jahrestemperatur unter $5^{\circ} \mathrm{C}$, Jahresniederschläge $800-1200 \mathrm{~mm}$, mehr als 240 Frosttage im Jahr, LAUER \& STIEHL 1973) ist die Bodenbildung so stark eingeschränkt, daß sich ein erheblicher Altersunterschied kaum auf die Eisenfreisetzung auswirkt. Unter weniger extremen Klimabedingungen (Höhen zwischen 2500 und $3800 \mathrm{~m}$ ü. NN) wirkt sich dagegen das höhere Alter der 2C-Aschen so deutlich auf die $\mathrm{Fe}_{\mathrm{d}}$-Gehalte aus, daß eine Unterscheidung zwischen 1C- und 2C-Aschen mit Hilfe dieser pedologischen Testgröße möglıch ist.

In 3C-Aschen ohne Bedeckung durch jüngere Aschen $\left(3 \mathrm{C}_{1}\right)$ ist die Bodenentwicklung gegenüber 2C-Aschen soweit fortgeschritten, daß die $\mathrm{Fe}_{\mathrm{d}}$-Gehalte auf 8,0-16,9\% stiegen sind. Die starke Streuung der Werte zeigt die differenzierende Wirkung der Klimavarianz in älteren Pyroklastika. Unbedeckte 3C-Aschen treten in Höhen über $3600 \mathrm{~m}$ nicht mehr auf, so daß Extremwerte, wie sie in Höhen über $4000 \mathrm{~m}$ zu erwarten sind, in der $3 C_{1}$-Verteilung nicht enthalten sind. 
Mit Hilfe der $\mathrm{Fe}_{\mathrm{d}}$-Werte lassen sich ohne Schwierigkeit $3 \mathrm{C}_{1^{-}}$von $1 \mathrm{C}$-Aschen unterscheiden. Auch die Unterscheidung von $3 \mathrm{C}_{1}$ und $2 \mathrm{C}$ ist mit Ausnahme eines schmalen Überschneidungsbereichs zwischen 8 und $9 \%$ möglich.

Die Form der Verteilung und die Tatsache, daß der größte Teil der Streuung auf die Wirkung der Klimavarianz zurückgeführt werden kann (MıEHLICH 1974), bestätigen, daß die in ihren Schwermineralverhältnissen stark streuuenden Proben von 3C einer Ausbruchsphase entstammen.

Die Bedeckung der 3C-Aschen durch Ablagerungen jüngerer Eruptionen (3C2), die meist $1-1,5 \mathrm{~m}$ erreicht, verlangsamt die Bodenbildung in den 3C-Aschen. Bei ähnlicher Streuung wie in $3 \mathrm{C}_{1}$, werden die $\mathrm{Fe}_{\mathrm{d}}$-Gehalte in $3 \mathrm{C}_{2}$ mit $3,0-10,9 \%$ so gering, daß eine Unterscheidung von $1 \mathrm{C}$ und $2 \mathrm{C}$ zu $3 \mathrm{C}_{2}$ nicht möglich ist. Ein grober Altersvergleich (Bodenbildung in 3C ohne Überdeckung max. ca. 7000 Jahre/Alter 2C-Aschen max. ca. 5000 Jahre) ergibt, daß die Eisenfreisetzung nach der Úberdeckung mit jüngeren Pyroklastika sehr stark eingeschränkt wurde.

\begin{tabular}{|c|c|c|c|c|c|c|c|c|c|c|c|c|c|c|c|c|c|c|c|}
\hline $3 \mathrm{C} / \mathrm{c}$ & & & & & 166 & $\begin{array}{l}177 \\
176\end{array}$ & & 228 & B 246 & 624 & & $\begin{array}{l}463 \\
244\end{array}$ & 443 & & $\begin{array}{l}412 \\
272 \\
\end{array}$ & & & 413 & $\begin{array}{l}545 \\
544\end{array}$ \\
\hline c & 4544 & 453 & $\begin{array}{r} \\
452 \\
431 \\
\end{array}$ & $\begin{array}{l}451 \\
435 \\
433 \\
432 \\
264 \\
257 \\
256 \\
255 \\
251 \\
248 \\
\end{array}$ & $\begin{array}{l} \\
\\
434 \\
263 \\
262 \\
261 \\
254 \\
103 \\
\end{array}$ & $\begin{array}{r} \\
253 \\
252 \\
240 \\
102 \\
\end{array}$ & $\begin{array}{r} \\
455 \\
105 \\
\end{array}$ & $\begin{array}{l}421 \\
249 \\
247 \\
106 \\
104 \\
\end{array}$ & & 178 & & 424 & & 422 & 265 & $\begin{array}{l}444 \\
423 \\
146\end{array}$ & $\begin{array}{l}273 \\
137\end{array}$ & $\begin{array}{l} \\
\\
464 \\
147 \\
136\end{array}$ & $\begin{array}{l} \\
446 \\
445 \\
414 \\
274 \\
159 \\
138 \\
135\end{array}$ \\
\hline $3 C / A$ & 497 & 531 & $\begin{array}{l}474 \\
473\end{array}$ & $\begin{array}{l}496 \\
495\end{array}$ & $\begin{array}{l}472 \\
471\end{array}$ & & $\begin{array}{l}494 \\
491 \\
353\end{array}$ & 493 & 3492 & & & & & & & & & & \\
\hline A & $\begin{array}{l}533 \\
532\end{array}$ & & & 354 & & & & & & & & & & & & & & & \\
\hline $\mathbf{S}$ & & 015 & $\begin{array}{l}187 \\
011\end{array}$ & $\begin{array}{l}016 \\
013 \\
012\end{array}$ & & 014 & & 181 & 182 & & 186 & 183 & & & & & & 185 & 184 \\
\hline G & & & $\begin{array}{l}215 \\
214\end{array}$ & & & & & & & & & & & & & & & & $\begin{array}{r}198 \\
.197\end{array}$ \\
\hline $\mathbf{M}$ & & & & & & & & & & & & 201 & & & & & $\begin{array}{l}203 \\
202\end{array}$ & & 204 \\
\hline $1 P$ & $\begin{array}{l}123 \\
092 \\
072 \\
063082\end{array}$ & $\begin{array}{l}073 \\
064 \\
042 \\
035\end{array}$ & $\begin{array}{l}222 \\
052\end{array}$ & & & & & & & & & & & & & & & & \\
\hline $2 P$ & $\begin{array}{l}213 \\
096 \\
094 \\
077 \\
069 \\
054 \\
044 \\
037\end{array}$ & 095 & $\begin{array}{l}225 \\
076\end{array}$ & & & $\begin{array}{l}233 \\
086\end{array}$ & & & & & & & & & & & & & \\
\hline \multirow[t]{2}{*}{$3 \mathbf{P}$} & & 058 & & & & & $\begin{array}{l}049 \\
027\end{array}$ & 158 & & & & & & & $\begin{array}{l}157 \\
026 \\
\end{array}$ & & $\begin{array}{l}145 \\
134\end{array}$ & & \\
\hline & 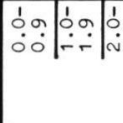 & \begin{tabular}{l|l} 
\\
vin
\end{tabular} & किं & $\left|\begin{array}{cc|}1 & 0 \\
\dot{x} & \dot{7}\end{array}\right|$ & 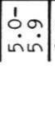 & $\left|\begin{array}{l}1 \\
\vdots \\
\dot{0} \\
\dot{0} \\
\end{array}\right|$ & 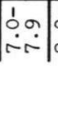 & 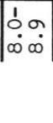 & केंक & '0 & $\left|\begin{array}{cc}1 & 0 \\
0 & 9 \\
\hdashline & - \\
\hdashline\end{array}\right|$ & 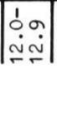 & $\begin{array}{l}1 \\
\text { bag } \\
\text { mim }\end{array}$ & 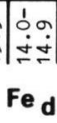 & 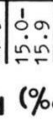 & 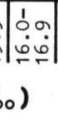 & |con & $\mid$\begin{tabular}{cc}
1 & 0 \\
0 & 0 \\
0 & $\infty$ \\
\hdashline & $\infty$
\end{tabular} & $\frac{0}{\alpha}$ \\
\hline
\end{tabular}

Tab. 3. Häufigkeitsverteilung des Gehalts an dithionitlöslichem Eisen $\left(\mathrm{Fe}_{\mathrm{d}}\right)$ in \%o der humusfreien Feinerde; Abkürzungen vgl. Abschnitt 1. 
Die Zwischenstellung der $\mathrm{Fe}_{\mathrm{d}}$-Gehalte für nicht sicher einzuordnende Proben $(1 \mathrm{C} / 2 \mathrm{C}$ und $2 \mathrm{C} / 3 \mathrm{C}$ ) zeigt, daß im Grenzbereich der Bimsverbreitung (1P, 2P) Aschen verschiedener Eruptionsphasen unmittelbar übereinanderliegen. Für den Nordrand der 2P-Verbreitung (Abb. 1) läßt sich ein Saum von ca. $5 \mathrm{~km}$ Breite nachweisen, in dem 2C über 3C-Aschen liegen. Für das Profil 02, das im Grenzbereich der geschlossenen Verbreitung von $1 \mathrm{P}$ und $2 \mathrm{P}$ liegt, kann die stratigraphische Abfolge $1 \mathrm{C}-2 \mathrm{C}-3 \mathrm{C}-3 \mathrm{P}$ wahrscheinlich gemacht werden.

Wie bereits aus den Vorüberlegungen zu vermuten, lassen sich die $\mathrm{Fe}_{\mathrm{d}}$-Gehalte für die älteren Pyroklastika (c) nicht zur Stratigraphie heranziehen (Tab. 3). Die Streuung der Werte reicht über den gesamten Bereich der im Untersuchungsgebiet auftretenden Gehalte, und die Verteilung ist deutlich 2-gipflig. Im Gegensatz zu 3C ist die Ursache für diese hohe Streuung vor allem in der primären Heterogenität der hier zusammengefaßten Pyroklastika zu suchen.

Dennoch ist eine Unterscheidung der älteren Pyroklastika c von 1C, 2C und 3C mit Hilfe des Aktivitätsgrades möglich. Der Aktivitätsgrad junger Andosole ist hoch und zeigt im Verlauf der Bodenentwicklung über mehrere Jahrtausende (1C bis $3 \mathrm{C}$ in Tab. 4) kaum altersabhängige Unterschiede, da die Kristallisation amorpher Eisenverbindungen durch den hohen Allophan- und Humusgehalt stark behindert wird (SCHwERTMANN \& a. 1968).

Gegen Ende der Andosolphase nehmen der Humus- und Allophangehalt stark ab, wodurch die Kristallisationshemmung vermindert wird. Die Aktivitätsgrade der älteren Pyroklastika sind dementsprechend gering (Tab. 5). Dabei wirkt sich die primäre Inhomogenität der Sedimente, die zu einer zweigipfligen Verteilung der $\mathrm{Fe}_{\mathrm{d}}$-Werte führte, nicht gleichermaßen auf den Kristallisationsgrad aus, da hier das relative Verhältnis $\mathrm{Fe}_{\mathrm{o}}: \mathrm{Fe}_{\mathrm{d}}$ bestimmt wird. Mit Ausnahme eines Überschneidungsbereichs zwischen 0,3 und 0,5 lassen sich daher mit Hilfe des Aktivitätsgrades die älteren Pyroklastika $\mathrm{c}$ von 1C, 2C und 3C unterscheiden. Dies ist besonders für $3 \mathrm{C} / \mathrm{c}$ wichtig, die sich bei fehlender 3P-Lage weder morphologisch noch schwermineralogisch eindeutig auseinanderhalten lassen.

\begin{tabular}{|c|c|c|c|c|c|c|c|c|}
\hline $1 C$ & 041 & 111 & $\begin{array}{l}211 \\
061071 \\
033 \quad 051\end{array}$ & $\begin{array}{l}231 \\
121 \\
032034\end{array}$ & 091 & 031 & & 081 \\
\hline $1 C / 2 C$ & & & 022 & & 021 & & & \\
\hline $2 \mathrm{C}$ & & & 065 & $\begin{array}{l}212 \\
067\end{array}$ & $\begin{array}{r}232 \\
066093\end{array}$ & $\begin{array}{l}2 \\
\frac{2}{3} 085074\end{array}$ & 4 & $\begin{array}{l}224 \\
223 \\
084 \\
083 \\
075 \\
053 \\
043 \\
036\end{array}$ \\
\hline $2 C / 3 C$ & & $\begin{array}{l}243 \\
023 \\
\end{array}$ & 242241 & & 161141 & $\begin{array}{l}162 \\
1151 \\
\end{array}$ & 191 & \\
\hline $3 C$ & $\begin{array}{ll} & \\
483 & 133 \\
132 & 025\end{array}$ & $\begin{array}{l} \\
411 \\
271 \\
237 \\
131 \quad 024 \\
\end{array}$ & $\begin{array}{rrr} & & \\
& & \\
& & 541 \\
& & 482 \\
543 & 542372 \\
462 & 371 & 236 \\
461 & 171 & 172 \\
\end{array}$ & 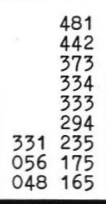 & $\begin{array}{ll} & \\
& \\
441 & \\
374 & 332 \\
174 & 173 \\
164 & 163 \\
078 & 142 \\
057 & 087 \\
\end{array}$ & $\begin{array}{lll} & & \\
& 351 & \\
156 & 352 \\
152 & 292 \\
2 & 144 & 227 \\
3 & 143 & 226 \\
3 & 055 & 196 \\
2 & 047 & 039 \\
7 & 030 & 038 \\
\end{array}$ & $\begin{array}{ll}2 & \\
2 & \\
2 & 291 \\
7 & 234 \\
6 & 193 \\
6 & 192 \\
9 & 155 \\
8 & 154 \\
8 & \\
\end{array}$ & $\begin{array}{l}293 \\
195 \\
194 \\
153 \\
046 \\
045 \\
\end{array}$ \\
\hline & 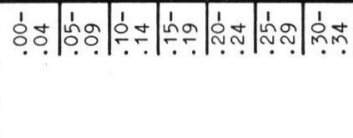 & 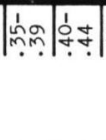 & 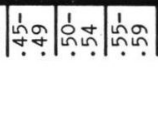 & $\mid$ & 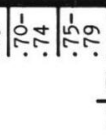 & 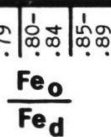 & & $\stackrel{n}{n}$ \\
\hline
\end{tabular}

Tab. 4. Häufigkeitsverteilung des Aktivitätsgrades ( $\mathrm{Fe}_{\mathrm{o}}: \mathrm{Fed}$ ); Abkürzungen vgl. Abschnitt 1. 
Bei Klimabedingungen, wie sie in der Sierra Nevada unterhalb $4000 \mathrm{~m}$ herrschen, ist anzunehmen, daß die Bodenentwicklung in Aschenlagen nach 10000-15000 Jahren so weit vorangeschritten ist, daß kein Allophan mehr nachweisbar ist. Es muß daher möglich sein - neben der Trennung durch die Eisenfraktionierung - auch durch die Prüfung auf Allophan die älteren Aschen c (ca. 25000 Jahre alt) von den jüngeren Aschen 1C-3C (400 bis ca. 9000 Jahre alt) zu trennen. Hierzu eignet sich der semiquantitative AllophanTest nach Fieldes \& Perrot (1966), der eine Einstufung in viel, wenig, kein Allophan erlaubt. Von 68 untersuchten Proben, die nach der Makromorphologie und der Eisenfraktionierung als Aschen 3C angesprochen wurden, zeigen 60 viel und 8 (meist 3C 2 Proben) wenig Allophan, während von 52 Proben, die als ältere Pyroklastika angesprochen wurden, 50 kein und 2 Proben wenig Allophan aufweisen. Von 15 Mischschichten haben 8 viel, 5 wenig und 2 kein Allophan. Der auch im Gelände durchführbare Allophan-Schnelltest erlaubt so eine einfache und sichere Unterscheidung der makroskopisch sehr ähnlichen 3C-Aschen von den älteren Pyroklastika c.

Auch für die bereits morphologisch leicht unterscheidbaren Substrate vulkanische Sande (A), fluviatil akkumulierte Sedimente (S), glazial aufbereitetes Sediment (G), Gesteins-

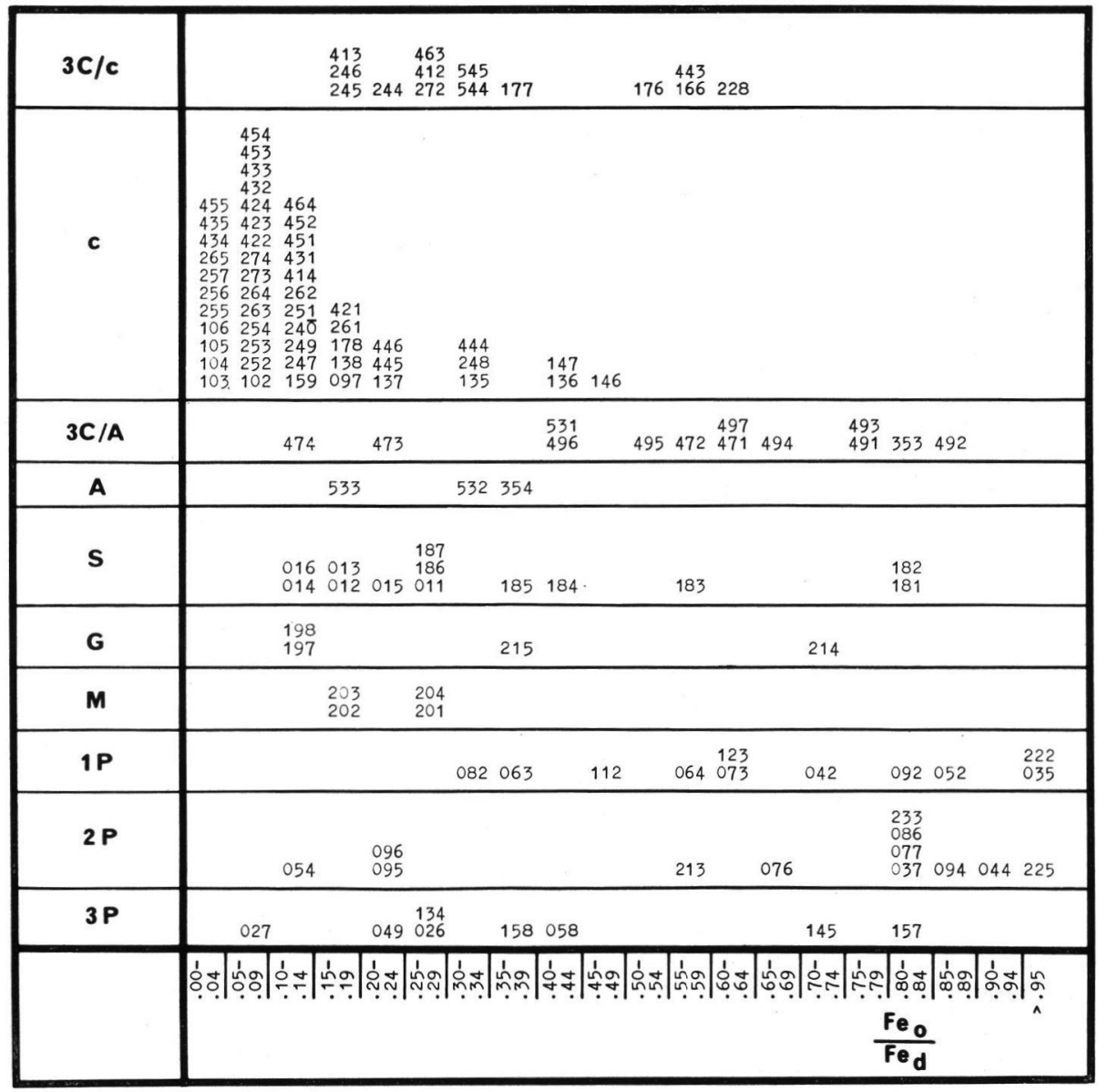

Tab. 5. Häufigkeitsverteilung des Aktivitätsgrades $\left(\mathrm{Fe}_{\mathrm{o}}: \mathrm{Fe}_{\mathrm{d}}\right)$; Abkürzungen vgl. Abschnitt 1. 
zersatz aus Phänoandesiten (M) sowie die Bimslagen 1P, 2P und 3P wurden Eisenfraktionierungen durchgeführt (Tab. 3 u. 5). Die Ergebnisse sollen hier kurz diskutiert werden, da sie Beispiele für die eingangs angeführten theoretischen Überlegungen darstellen.

Die Eisenfreisetzung in Böden aus vulkanischen Sanden des Tlaloc/Telapón-Gebiets ist altersabhängig deutlich geringer, als in den jüngeren Aschen des Popocatépetl, da die primäre Zusammensetzung eisenärmer und die Verwitterungsrate durch die grobe Körnung verringert ist. Dem entsprechen $\mathrm{Fe}_{\mathrm{d}}$-Gehalte zwischen $\mathrm{O}$ und $5 \%$. Weiterhin ist anzunehmen, daß die heute oberflächlich anstehenden vulkanischen Sande erst vor relativ kurzer Zeit erosiv freigelegt wurden, so daß die $\mathrm{Fe}_{\mathrm{d}}$-Werte nicht die Eisenfreisetzung während des gesamten Zeitraums nach der Ablagerung (ca. 22000 Jahre) repräsentieren. Unter diesen Voraussetzungen lassen sich pedologische Methoden nicht zur Stratigraphie heranziehen.

Die $\mathrm{Fe}_{\mathrm{d}}$-Gehalte in fluviatilen Sedimenten spiegeln die Herkunft ihrer Ablagerungen wider. Die $\mathrm{Fe}_{\mathrm{d}}$-Gehalte in Profil 18 streuen über nahezu den gesamten Bereich; sie zeigen, daß dieses Profil aus dünnen Lagen sehr unterschiedlich vorverwitterter Pyroklastika aufgebaut ist, während das Profil 1 bei geringer Streuung ein in Profiltiefe sehr ähnliche Zusammensetzung der fluviatilen Sedimente erkennen läßt. Auch in glazial aufbereitetem Material $(\mathrm{G})$ hängen die $\mathrm{Fe}_{\mathrm{d}}$-Gehalte stark von der Zusammensetzung der transportierten Gesteine ab. Wie bei den fluviatil akkumulierten Sedimenten sind ihre Fe $\mathrm{d}_{\mathrm{d}}$-Gehalte kein $\mathrm{Maß}$ für die altersabhängig autochthone Bodenbildung, da teilweise schon vorverwittertes Material in den glazialen Transport einbezogen ist (z. B. Gesteinszersatz aus Phänoandesiten (M) in Profil 19).

Die intensiv rote Farbe des Gesteinszersatzes aus Phänoandesiten (M) deutet bereits auf einen hohen Gehalt an $\mathrm{Fe}_{\mathrm{d}}$, der jedoch keinesfalls zur Relativdatierung verwandt werden kann, da es sich hierbei um eine reliktische Bodenbildung in einem durch glaziale Vorgänge im Bereich der Iztaccihuatl freigelegten, sehr viel älteren Gesteinszersatz handelt. Die mit der Tiefe zunehmenden Gehalte an $\mathrm{Fe}_{\mathrm{d}}$ im Profil 20 sind vermutlich auf die abnehmenden Beimengungen jüngerer Aschen zurückzuführen.

Die Auswirkung unterschiedlicher Formen von Pyroklastika auf die Bodenbildung läßt sich aus dem Vergleich der vom Chemismus her sehr ähnlichen Substrate 1P, 2P, 3P mit den entsprechenden Feinaschelagen 1C, 2C und 3C zeigen. Die Verwitterung der Bimslage beschränkt sich auf eine altersabhängig dicker werdende Verwitterungsrinde der Bimspartikel; von einer Bodenbildung kann kaum gesprochen werden. Die an Feinerdeproben $(\phi<2 \mathrm{~mm})$ gewonnenen Ergebnisse täuschen zu hohe Werte vor, da diese Fraktion teilweise nur $10 \%$ der überwiegend feinkiesigen Bimslagen ausmacht.

Insgesamt zeigen die Ergebnisse, daß es möglich ist, verschieden alte Pyroklastika durch die Analyse altersabhängiger Bodenentwicklung zu unterscheiden. Der erfolgreiche Einsatz dieser Methode ist jedoch an die sorgfältige Überprüfung einer größeren Zahl von Voraussetzungen geknüpt.

\section{Literaturverzeich nis}

Aeppl, H.: Barroböden und Tepetate. - Diss., Gießen 1973.

Blume, H.-P. \& Schwertmann, U.: Genetic Evaluation of Profile Distribution of Aluminium, Iron and Manganese Oxides. - Soil Sci. Soc. Amer. Proc. 33, 438-444, Madison 1969.

Connwall, J. W.: Estratigrafía de los depósitos terrestres volcánicos y de los suelos entre Chalco y Puebla. - Bol. INAH 32, 25-29, Mexiko 1968.

Ern, H. \& Miehlich, G.: Diferencia de Vegetación y Suelos Dependientes del Relieve en un "Valle Cerrado“ de la Sierra Nevada, México. - Comunicaciones Proyecto Puebla-Tlaxcala 5, 9-14, Puebla 1972. 
Fieldes, M. \& Perrot, K. W.: The Nature of Allophane in Soils, 3. - N. Z. Jl Sci. 9, 623-629, 1966.

FreChEN, J.: Die Herkunft der spätglazialen Bimstuffe in mittel- und süddeutschen Mooren. Geol. Jb. 67, 209-230, Hannover 1952.

HeINE, K.: Zur Glazialmorphologie und präkeramischen Archäologie des mexikanischen Hochlandes während des Spätglazials (Wisconsin) und Holozäns. - Erdkunde 27, 161-180, Bonn 1973.

Heine, K. \& Heide-WeISE, H.: Secuencias de erupciones en el volcán de la Malinche y en la Sierra Nevada durante los últimos 40.000 años. - Comunicaciones Proyecto Puebla-Tlaxcala 7, 7-8, Puebla 1973.

Heine, K. \& Schönhals, E.: Entstehung und Alter der „toba“-Sedimente in Mexiko. - Eiszeitalter u. Gegenwart 23/24, 201-215, OOhringen 1973.

Jackson, M. L.: Soil Chemical Analysis. — Englewood Cliffs 1958.

Kaltofen, R.: Tabellenbuch der Chemie. Leipzig 1973.

KNEIB, W.: Versuch einer bodengeographischen Gliederung der Sierra Nevada de México. - Unveröff. Diplomarbeit, Hamburg 1973.

Kneib, W., Miehlich, G. \& Zöttr, H. W.: Clasificación Regional de los suelos de la Sierra Nevada de México. - Comunicaciones Proyecto Puebla-Tlaxcala 7, 11-13, Puebla 1973.

Lauer, W. \& Stiehl, E.: Hygrothermische Klimatypen im Raum Puebla-Tlaxcala (Mexiko). Erdkunde 27, 230-234, Bonn 1973.

Menra, O. P. \& Jackson, M. L.: Iron Oxide Removal from Soils and Clays by a DithioniteCitrate System buffered with Sodium Bicarbonate. - Clays and Clay Minerals, London 1960.

MeYer, B. \& SAKR, R.: Menge, Sitz und Verteilung der extrahierbaren Fe-, Al-, $\mathrm{SiO}_{2}-$ und HumusAnteile und ihr Einfluß auf die Austausch-Eigenschaften von typischen sauren Lockerbraunerden. - Göttinger Bodenkundl. Berichte 14, 49-83, Göttingen 1970.

Merer, J,: Stratigraphie der Bimskiese und -aschen des Coatepeque-Vulkans im westlichen El Salvador (Mittelamerika). - N. Jb. Geol. Paläont. Abh. 119, 215-246, Stuttgart 1964.

MienLich, G.: Klima- und altersabhängige Bodenentwicklung von Vulkanascheböden der Sierra Nevada de México. - Mitteilungen d. Dtsch. Bodenkundl. Ges. 18, 360-369, Göttingen 1974.

Miehlich, G., KNeIb, W. \& HeIDe-Weise, H.: Los materiales de partida de los suelos en la región Sierra Nevada, México. - Comunicaciones Proyecto Puebla-Tlaxcala, in Vorbereitung.

Mooser, F. H.: Tefracronología de la Cuenca de México para los últimos treinta mil años. - Bol. INAH 30, 12-15, Mexiko 1967.

Schwertmann, U.: Differenzierung der Eisenoxide des Bodens durch Extraktion mit Ammoniumoxalat-Lösung. - Z. Pflanzenern. Düng. Bodenkunde 105, 194-202, Weinheim/Bergstr. 1964.

Schwertmann, U., Fischer, W. R. \& Papendorf, H.: The Influence of Organic Compounds on the Formation of Iron Oxides. - Transactions 9th Intern. Congr. of Soil Sci. I, 645-655, Sidney 1968.

WeyL, R.: Beiträge zur Geologie El Salvadors. IV. Die Bimsaschen in der Umgebung San Salvadors. - Neues Jb. Geol. Paläontol. Mh. 2, 49-70, Stuttgart 1954.

White, S. E.: El Iztaccihuatl. - INAH, Serie Investigaciones 6, Mexiko 1962.

Yarza de la Torre: Volcanes de México. Mexiko 1971.

Manuskript eingeg. 13. 5. 1974.

Anschrift des Verf.: Dr. Günter Miehlich, Ordinariat für Bodenkunde der Universität Hamburg, 2057 Reinbek, Schloß. 